\title{
ZERO-DIVISOR GRAPHS WITH RESPECT TO PRIMAL AND WEAKLY PRIMAL IDEALS
}

\author{
Shahabaddin Ebrahimi Atani and Ahamd Yousefian Darani
}

ABStRaCt. We consider zero-divisor graphs with respect to primal, nonprimal, weakly prime and weakly primal ideals of a commutative ring $R$ with non-zero identity. We investigate the interplay between the ringtheoretic properties of $R$ and the graph-theoretic properties of $\Gamma_{I}(R)$ for some ideal $I$ of $R$. Also we show that the zero-divisor graph with respect to primal ideals commutes by localization.

\section{Introduction}

The idea of associating a graph with the zero-divisors of a commutative ring was introduced by Beck in 1988, where the author talked about the colorings of such graphs. By the definition he gave, every element of the ring $R$ was a vertex in the graph, and two vertices $x, y$ were adjacent if and only if $x y=0$ ([4]). We adopt the approach used by D. F. Anderson and P. S. Livingston ([2]) and consider only non-zero zero-divisors as vertices of the graph. The zero-divisor graph of a commutative ring has been studied extensively by several authors (see, for example, $[2,4,5,10,11,12]$ ).

Redmond [13] introduced the definition of the zero-divisor graph with respect to an ideal. Let $I$ be an ideal of a ring $R$. The zero-divisor graph of $R$ with respect to $I$ is an undirected graph, denoted by $\Gamma_{I}(R)$, with vertices $\{x \in R-I: x y \in I$ for some $y \in R-I\}$ where distinct vertices $x$ and $y$ are adjacent if and only if $x y \in I$. Therefore, if $I=0$ then $\Gamma_{I}(R)=\Gamma(R)$, and $I$ is a non-zero prime ideal if and only if $\Gamma_{I}(R)=\emptyset([13])$. The graphs $\Gamma_{I}(R)$ and $\Gamma(R / I)$ are different graphs. If fact for $x, y \in R \backslash I$, if $x+I$ is adjacent to $y+I$ in $\Gamma(R / I)$, then $x$ and $y$ are adjacent in $\Gamma_{I}(R)$; while the converse is true only when $x+I \neq y+I$ (see [13, Theorem 2.5]). Hence the study of the graph $\Gamma_{I}(R)$ is worthy of study. There are many basic open questions concerning the zerodivisor graph with respect to an ideal. One of the essential questions is whether a zero-divisor graph with respect to an ideal commutes with localization, and in this case, what are the relations between the diameters (resp. girths) of such graphs. We give a condition giving an affirmative answer to these questions.

Received July 5, 2007.

2000 Mathematics Subject Classification. 05C75, 13A15.

Key words and phrases. zero-divisor graph, primal, weakly primal. 
For the sake of completeness, we state some definitions and notations used throughout. We will use $R$ to denote a commutative ring with identity. We use $Z(R)$ to denote the set of zero-divisors of $R$; we use $Z(R)^{*}$ to denote the set of non-zero zero-divisors of $R$. By the zero-divisor graph of $R$, denoted $\Gamma(R)$, we mean the graph whose vertices are the non-zero zero-divisors of $R$, and for distinct $x, y \in Z(R)^{*}$, there is an edge connecting $x$ and $y$ if and only if $x y=0$. A graph is said to be connected if there exists a path between any two distinct vertices. For two distinct vertices $a$ and $b$ in a graph $G$, the distance between $a$ and $b$, denoted $\mathrm{d}(a, b)$, is the length of the shortest path connecting $a$ and $b$, if such a path exists; otherwise, $\mathrm{d}(a, b)=\infty$. The diameter of a connected graph is the supremum of the distances between vertices. We will use the notation $\operatorname{diam}(G)$ to denote the diameter of the graph of $G$. A graph is complete if it is connected with diameter 0 . A bipartite graph is a graph $G$ whose vertex set $V$ can be partitioned into two non-empty sets $V_{1}$ and $V_{2}$ in such a way that every edge of $G$ joins a vertex in $V_{1}$ to a vertex in $V_{2}$. The girth of a graph $G$, denoted $\operatorname{gr}(G)$, is the length of a shortest cycle in $G$, provided $G$ contains a cycle; otherwise, $\operatorname{gr}(G)=\infty$.

An ideal $I$ of $R$ is called a radical ideal if $I=\sqrt{I}$. A ring $R$ is called reduced if it contains no non-zero nilpotent elements. It is easy to see that $I$ is a radical ideal of $R$ if and only if $R / I$ is a reduced ring. Denote by $\operatorname{Min}(I)$ the set of minimal prime ideals of $R$ containing $I$.

Let $R$ be a commutative ring. A proper ideal $P$ of $R$ is said to be weakly prime if $0 \neq a b \in P$ implies that $a \in P$ or $b \in P$ ([3]). However, since 0 is always weakly prime (by definition), a weakly prime ideal need not be prime. We recall from [8] and [7], that an element $a \in R$ is called prime (resp. weakly prime) to an ideal $I$ of $R$ if $r a \in I$ (resp. $0 \neq r a \in I$ ) (where $r \in R$ ) implies that $r \in I$. Denote by $S(I)$ (resp. $w(I)$ ) the set of elements of $R$ that are not prime (resp. are not weakly prime) to $I$. A proper ideal $I$ of $R$ is said to be primal if $S(I)$ forms an ideal (so 0 is not necessarily primal); this ideal is always a prime ideal, called the adjoint ideal $P$ of $I$. In this case we also say that $I$ is a $P$-primal ideal of $R([8])$. Not that if $r \in R$ and $a \in S(I)$, then clearly $r a \in S(I)$. So what we require for $I$ being primal is that if $a$ and $b$ are not prime to $I$, then their difference is also not prime to $I$. For example assume that $R=\mathbb{Z}$ the ring of integers and let $I=6 \mathbb{Z}$. Then 2 and 3 are not prime to $I$ but $3-2$ is prime to $I$, so $I$ is not primal. A ring $R$ is said to be primal if the zero ideal is a primal ideal of $R$. Also, a proper ideal $I$ of $R$ is called weakly primal if the set $P=w(I) \cup\{0\}$ forms an ideal; this ideal is always a weakly prime ideal ([7, Proposition 4$])$. In this case we also say that $I$ is a $P$-weakly primal ideal. If $R$ is not an integral domain, then 0 is a 0 -weakly primal ideal of $R$ (by definition), so a weakly primal ideal need not be primal.

Assume that $S$ is a multiplicatively closed subset of a $\operatorname{ring} R, X$ a non-empty subset of $R$ and $I$ an ideal of $R$. Set $S^{-1} X=\{a / s \mid a \in X, s \in S\} \subseteq S^{-1} R$. We say that a zero-divisor graph with respect to $I$ commutes with localization 
if $S^{-1}\left(\Gamma_{I}(R)\right)=\Gamma_{S^{-1} I}\left(S^{-1} R\right)$. The main goal of this paper is to show that if $I$ is primal (resp. weakly primal), then $\Gamma_{I}(R)$ commutes with localization. Surely, there is much more work to be done.

Here is a brief summary of our paper. In Section 2, it is shown that (Theorem 2.5), if $I$ and $J$ are $P$-primal ideals of $R$, then $\Gamma_{I}(R)=\Gamma_{J}(R)$ if and only if $I=J$. It is proved that (Theorem 2.8) if $I$ is a primal ideal of a Noetherian ring $R$, then $\operatorname{diam}(\Gamma(R / I)) \leq 2$. In Theorems 2.16 and 2.18 (resp. Theorems 4.7 and 4.8 ), it is shown that, if $S$ is a multiplicatively closed subset of $R$ which consists of regular elements of $R$ and $I$ is a $P$-primal (resp. $P$-weakly primal) ideal of $R$ with $P \cap S=\emptyset$, then $\operatorname{diam}\left(\Gamma_{I}(R)\right)=\operatorname{diam}\left(\Gamma_{S^{-1} I}\left(S^{-1} R\right)\right)$ and $\operatorname{gr}\left(\Gamma_{I}(R)\right)=\operatorname{gr}\left(\Gamma_{S^{-1} I}\left(S^{-1} R\right)\right)$.

In Section 3, it is proved that, if $I=\sqrt{I}$ is not a primal ideal of $R$ with $|\operatorname{Min}(I)| \geq 3$, then $\operatorname{diam}\left(\Gamma_{I}(R)\right)=3$ (Theorem 3.3). Also, it is shown that, if $I \neq \sqrt{I}$ is not a primal ideal of $R$, then $\operatorname{diam}\left(\Gamma_{I}(R)\right)=3$ (Theorem 3.7).

In Section 4 , we study the zero-divisor graph with respect to a weakly primal ideal. We put $Z_{I}(R)=\{r \in R-I: r a=0$ for some $a \in R-I\}$ where $I$ is an ideal of $R$. It is proved that (Theorem 4.3), if $I$ is an ideal of a ring $R$ and $P$ is a weakly prime ideal with $w(I) \subseteq P$ and $(P-I) \cap Z_{I}(R)=\emptyset$, then $\Gamma_{I}(R)=(P-I) \cup Z_{I}(R)$ if and only if $I$ is a $P$-weakly primal ideal of $R$. It is shown that (Theorem 4.4) if $I$ is a weakly prime ideal of $R$, then $\Gamma_{I}(R)=Z_{I}(R)$. In particular, $\Gamma_{I}(R)$ is a subgraph of $\Gamma(R)$.

\section{Primal ideals}

In this section, we will investigate the ideal-based zero-divisor graph with respect to primal ideals. The class of primal ideals is a large class. For example all primary ideals and irreducible ideals are primal. So the structure of zerodivisor graphs with respect to primal ideals is worthy of study. Our starting point is the following lemma:

Lemma 2.1. Let $I$ be a proper ideal of a ring $R$. Then the following hold:

(i) $I \subseteq S(I)$.

(ii) $\Gamma_{I}(R)=S(I)-I$. In particular, $\Gamma_{I}(R) \cup I=S(I)$.

(iii) If $I$ is a radical ideal of $R$, then $S(I)=\bigcup_{P \in \operatorname{Min}(I)} P$.

Proof. (i) Let $x \in I$. As $x .1_{R} \in I$ with $1_{R} \notin I$, we must have $x$ is not prime to $I$; hence $I \subseteq S(I)$.

(ii) Let $r \in \Gamma_{I}(R)$. Then $r \notin I$ and $r x \in I$ for some $x \notin I$, so $r$ is not prime to $I$; hence $r \in S(I)-I$. Thus $\Gamma_{I}(R) \subseteq S(I)-I$. For the other containment, assume that $a \in S(I)-I$. As $a$ is not prime to $I$, there exists $y \notin I$ such that $a y \in I$. Then $a \in \Gamma_{I}(R)$, so we have equality.

(iii) Let $x \in S(I)$. Thus we may assume that $x \notin I$, so $x \in \Gamma_{I}(R)$. Then $x y \in I$ for some $y \in R-I$, so $\left(0:_{R / I} x+I\right) \neq 0$; hence $x+I \in P / I$ for some minimal prime ideal $P / I$ of $R / I$ by [9, Corollary 2.3]. Therefore, $P \in \operatorname{Min}(I)$ and $x \in P$ gives $S(I) \subseteq \bigcup_{P \in \operatorname{Min}(I)} P$. Conversely, assume that $x \in P$ for some 
minimal prime ideal $P$ of $I$. If $x \in I$, then $x \in S(I)$ by (i). So we can assume that $x \notin I$. By [9, Theorem 2.1], there exist $y \notin P$ and a positive integer $n$ such that $y x^{n} \in I$ but $y x^{n-1} \notin I$. This implies that $x \in \Gamma_{I}(R)$, so we have equality.

Proposition 2.2. Let $I$ and $P$ be ideals of a ring $R$ with $I \subseteq P$. Then $I$ is a $P$-primal ideal of $R$ if and only if $\Gamma_{I}(R)=P-I$.

Proof. If $I$ is a $P$-primal ideal of $R$, then $\Gamma_{I}(R)=S(I)-I=P-I$ by Lemma 2.1. Conversely, assume that $\Gamma_{I}(R)=P-I$. It suffices to show that $P$ is exactly the set of elements of $R$ that are not prime to $I$. First, suppose that $c \in P$. Since every element of $I$ is not prime to $I$, we can assume that $c \in P-I=\Gamma_{I}(R)$. Then there exists $z \notin I$ such that $c z \in I$, so $c$ is not prime to $I$. Next suppose that $s$ is not prime to $I$. If $s \in I$, then $s \in P$. If $s \notin I$, then there is an element $t \notin I$ such that $s t \in I$, so $s \in \Gamma_{I}(R)=P-I \subseteq P$. Thus $I$ is a $P$-primal ideal of $R$.

Theorem 2.3. Let $I$ be an ideal of a ring $R$. Then $I$ is a primal ideal of $R$ if and only if $\Gamma_{I}(R) \cup I$ is an (prime) ideal of $R$.

Proof. This follows from Proposition 2.2.

Examples 2.4. (1) Let $R=\mathbb{Z}_{16}, I=(\overline{4})$ and $P=(\overline{2})$. It is easy to check that $I$ is a $P$-primal ideal of $R$. Then $\Gamma_{I}(R)=P-I=\{\overline{2}, \overline{6}, \overline{10}, \overline{1} 4\}$ by Proposition 2.2.

(2) Let $R=\mathbb{Z}, I=9 \mathbb{Z}$ and $P=3 \mathbb{Z}$. Then, $I$ is a $P$-primary and hence a $P$ primal ideal of $R$ by [7, Lemma 19]. Hence $\Gamma_{I}(R)=3 \mathbb{Z}-9 \mathbb{Z}$ by Proposition 2.2.

(3) Let $R=Q[x, y]$ the ring of polynomials in $x$ and $y$ with rational numbers for their coefficients. Then $I=\left(x^{2}, x y\right)$ is a primal ideal with adjoint ideal $P=(x, y)([8])$. Hence $\Gamma_{I}(R)=(x, y)-\left(x^{2}, x y\right)$ by Proposition 2.2 .

Let $I, J$ be ideals of a ring $R$. It is natural to ask whether $\Gamma_{I}(R)=\Gamma_{J}(R)$ implies $I=J$ ? In this case, we have the following theorem:

Theorem 2.5. Let $I$ and $J$ be P-primal ideals of a ring $R$. Then $\Gamma_{I}(R)=$ $\Gamma_{J}(R)$ if and only if $I=J$.

Proof. By Lemma 2.1, $I \subseteq P$ and $J \subseteq P$. It then follows from Proposition 2.2 that $\Gamma_{I}(R)=\Gamma_{J}(R)$ if and only if $P-I=P-J$; and this holds if and only if $I=J$.

Theorem 2.6. Let $I$ be an ideal of a ring $R$. Then $I$ is primary if and only if $\Gamma_{I}(R)=\sqrt{I}-I$.

Proof. If $I$ is primary, then $I$ is a $\sqrt{I}$-primal ideal of $R$ by [7, Lemma 19], so Proposition 2.2 gives $\Gamma_{I}(R)=\sqrt{I}-I$. Conversely, suppose that $a, b \in R$ are such that $a b \in I$ but $a \notin I$ and $b \notin \sqrt{I}$ (so $b \notin I$ ). Then $b \in \Gamma_{I}(R)=\sqrt{I}-I$, which is a contradiction. Thus $I$ is primary. 
Theorem 2.7. Let $I$ be an ideal of a commutative ring $R$. Then:

(1) 0 is a primal ideal of $R$ if and only $Z(R)$ is an (prime) ideal of $R$.

(2) $I$ is a primal ideal of $R$ if and only if $Z(R / I)$ is an ideal of $R / I$.

Proof. (1) It is easy to show that $S(0)=Z(R)$. Now the result follows from the definition.

(2) It follows from (1).

Let $I$ be an ideal of $R$. It is shown in [2, Theorem 2.3] and [13, Theorem 2.4] that $\operatorname{diam}(\Gamma(R / I)) \leq 3$ and $\operatorname{diam}\left(\Gamma_{I}(R)\right) \leq 3$, but for a primal ideal we have the following results:

Theorem 2.8. Let $R$ be a Noetherian ring. If $I$ is a primal ideal of $R$, then $\operatorname{diam}(\Gamma(R / I)) \leq 2$.

Proof. Let $I$ be a $P$-primal ideal of $R$. Then $\Gamma(R / I) \cup\{0+I\}=P / I$ is a prime ideal of $R / I$ by Theorem 2.7 and by [14, Corollary 9.36], $P / I=\bigcup_{\bar{Q} \in \operatorname{Ass}(R / I)} \bar{Q}$; hence $P / I \in \operatorname{Ass}(R / I)$. Therefore, $P / I=\left(0:_{R / I} \bar{a}\right)$ for some $\bar{a} \in \Gamma(R / I)$. It follows that $\operatorname{diam}(\Gamma(R / I)) \leq 2$.

Let $R$ be a principal ideal domain and let $p$ be an irreducible element of $R$. Then $R p^{t}$ is a primary ideal of $R$ for every positive integer $t$. So $\operatorname{Nil}\left(R / R p^{t}\right)=Z\left(R / R p^{t}\right)$. Therefore $\operatorname{diam}\left(\Gamma\left(R / R p^{t}\right)\right) \leq 2$ by [10, Lemma 2.3]. Using Theorem 2.8, we give another proof for this result.

Corollary 2.9. Let $R$ be a principal ideal domain, and let $p$ be an irreducible element of $R$. Then for every positive integer $t, \operatorname{diam}\left(\Gamma\left(R / R p^{t}\right)\right) \leq 2$. In particular, diam $\left(\Gamma\left(\mathbb{Z}_{p^{t}}\right)\right) \leq 2$ for every prime number $p$.

Proof. Since $I=R p^{t}$ is primary, we must have $I$ is a primal ideal of $R$ by [7, Lemma 19]. Now the assertion follows from Theorem 2.8 .

Example 2.10. Let $R=\mathbb{Z}$ and $I=18 \mathbb{Z}$. Then $I$ is not a primal ideal of $R$ since 2 and 3 are not prime to $I$, but $3-2=1$ is prime to $I$. Consider the elements $\overline{2}$ and $\overline{3}$ in $R / I$. As $\overline{2} . \overline{3} \neq 0$ we have $d(\overline{2}, \overline{3}) \neq 1$. If there is a vertex $\bar{a}$ in $\Gamma(R / I)$ such the $\overline{2}-\bar{a}-\overline{3}$ is a path, then $\bar{a}=0$ which is a contradiction. Hence $d(\overline{2}, \overline{3}) \neq 2$. Thus $\operatorname{diam}(\Gamma(R / I))=3$. Therefore, the condition " $I$ is a primal ideal of $R$ " is not superficial in the Theorem 2.8 .

Let $R$ be a Noetherian ring and assume that $Q(R)$, the total quotient ring of $R$, is local. In this case, $Z(Q(R))=\operatorname{ann}(x)$ for some $0 \neq x \in Q(R)$. This implies that $\operatorname{diam}(\Gamma(Q(R)) \leq 2$. On the other hand, $\Gamma(Q(R))$ is isomorphic to $\Gamma(R)$ by $[1$, Theorem 2.2$]$. So $\operatorname{diam}(\Gamma(R)) \leq 2$. However this fact may be proved as the following theorem.

Theorem 2.11. Assume that $R$ is a Noetherian ring let $Q(R)$, the total quotient ring of $R$ a local ring. Then $\operatorname{diam}(\Gamma(R)) \leq 2$. 
Proof. Assume that $S$ is the set of non-zero-divisors of $R / I$ and let $M$ be the unique maximal ideal of $Q(R)$. Then there exists a prime ideal $P$ of $R$ such that $P \cap S=\emptyset$ and $M=S^{-1} P$. First we show that $P=Z(R)$. As $P \cap S=\emptyset$, we have $S \subseteq R-P$. For every $a \in R-P$, we have $a / 1 \notin S^{-1} P=M$. Hence $a / 1$ is a unit in $Q(R)$. Thus $a \notin Z(R)$, that is $R-P \subseteq S$. Therefore $P=Z(R)$. So 0 is a $P$-primal ideal of $R$ by Theorem 2.7. Now the result follows from Theorem 2.8 .

Lemma 2.12. Let $I$ be a radical ideal of a ring $R$. Then $\operatorname{diam}(\Gamma(R / I))=$ $\operatorname{diam}\left(\Gamma_{I}(R)\right)$.

Proof. This follows immediately from [5, Lemma 2.1 and Proposition 2.2].

Corollary 2.13. Let $I$ be a primal radical ideal of a Noetherian ring $R$. Then $\operatorname{diam}\left(\Gamma_{I}(R)\right) \leq 2$.

Proof. This follows from Theorem 2.8 and Lemma 2.12.

We shall require the following proposition, and its proof is a modification of those in [6, Lemma 2.11 and Proposition 2.14], but we give the details for convenience.

Proposition 2.14. Assume that $S$ is a multiplicatively closed subset of a ring $R$ and let $I$ be a $P$-primal ideal of $R$ with $P \cap S=\emptyset$. Then the following hold:

(i) If $a / s \in S^{-1} I$, then $a \in I$.

(ii) $S^{-1} I$ is a $S^{-1} P$-primal ideal of $S^{-1} R$.

Proof. (i) Suppose that $a / s \in S^{-1} I$, but $a \notin I$. Then there are elements $a^{\prime} \in I$ and $t \in S$ such that $a / s=a^{\prime} / t$, so $u t a=u s a^{\prime} \in I$ for some $u \in S$. It follows that $u t$ is not prime to $I$; hence $u t \in P \cap S$ which is a contradiction, as needed.

(ii) Clearly, $S^{-1} P$ is a prime ideal of $S^{-1} R$. It is enough to show that $S^{-1} P$ is exactly the set of elements of $S^{-1} R$ which are not prime to $S^{-1} I$. Let $r / s \in S^{-1} P$. Then $r$ is not prime to $I$, so there exists $c \in R-I$ with $r c \in I$. Since $P \cap S=\emptyset$, we get $s c \notin I$; hence $(s c) / 1 \notin S^{-1} I$ by (i). As $(r / s)(s c) / 1 \in S^{-1} I$, we must have $r / s$ is not prime to $S^{-1} I$. Now assume that $r / s$ is not prime to $S^{-1} I$. Then there exists $d / t \notin S^{-1} I$ with $(r / s)(d / t) \in S^{-1} I$; hence $r d \in I$ by (i). Since $d \notin I$, it follows that $r$ is not prime to $I$. Thus $r \in P$, and hence $r / s \in S^{-1} P$, as required.

Proposition 2.15. Assume that $S$ is a multiplicatively closed subset of a ring $R$ and let $I$ be a P-primal ideal of $R$ with $P \cap S=\emptyset$. Then $S^{-1}\left(\Gamma_{I}(R)\right)=$ $\Gamma_{S^{-1} I}\left(S^{-1} R\right)$.

Proof. By Proposition 2.2 and Proposition 2.14, we must have $\Gamma_{S^{-1} I}\left(S^{-1} R\right)=$ $S^{-1} P-S^{-1} I$. It suffices to show that $S^{-1}(P-I)=S^{-1} P-S^{-1} I$. First, suppose that $a / s \in S^{-1} P-S^{-1} I$. Then $a / s \notin S^{-1} I$ (so $\left.a \notin I\right)$ and $(a / s)(b / t)=$ $a b / s t \in S^{-1} I$ for some $b / t \notin S^{-1} I$ (so $b \notin I$ ), so $a b \in I$ by Proposition 2.14(i); hence $a$ is not prime to $I$. It follows that $a / s \in S^{-1}(P-I)$. Thus $S^{-1} P-$ 
$S^{-1} I \subseteq S^{-1}(P-I)$. Next, assume that $a / s \in S^{-1}(P-I)$. Then $a \in P-I$ implies that $a b \in I$ for some $b \notin I$ by Proposition 2.2, so by Proposition 2.14(i), $b / 1 \notin S^{-1} I$. Now $(a / s)(b / 1)=a b / s \in S^{-1} I$ gives $a / s$ is not prime to $S^{-1} I$, so $a / s \in S^{-1} P-S^{-1} I$; hence we have equality.

Theorem 2.16. Assume that $S$ is a multiplicatively closed subset of a ring $R$ which consists of regular elements of $R$ and let $I$ be a $P$-primal ideal of $R$ with $P \cap S=\emptyset$. Then $\operatorname{diam}\left(\Gamma_{I}(R)\right)=\operatorname{diam}\left(\Gamma_{S^{-1}}\left(S^{-1} R\right)\right)$.

Proof. Suppose that $\operatorname{diam}\left(\Gamma_{I}(R)\right)=1$. For every distinct vertices $a / s, b / t$ of $\Gamma_{S^{-1} I}\left(S^{-1} R\right)$ ), Proposition 2.15 gives $a$ and $b$ are distinct elements of $\Gamma_{I}(R)$, so $a b \in I$; hence $(a / s)(b / t) \in S^{-1} I$. Thus $\operatorname{diam}\left(\Gamma_{S^{-1} I}\left(S^{-1} R\right)\right)=1$. If $\operatorname{diam}\left(\Gamma_{S^{-1} I}\left(S^{-1} R\right)\right)=1$, then for every distinct vertices $a, b$ of $\Gamma_{I}(R)=P-I$, we have the distinct vertices $a / 1, b / 1 \in S^{-1}(P-I)=\Gamma_{S^{-1} I}\left(S^{-1} R\right)$ by Proposition 2.15 (since if $a / 1=b / 1$, then $t a=t b$ for some $t \in S$; this implies that $a=b$ which is a contradiction), so $(a / 1)(b / 1) \in S^{-1} I$. It follows from Proposition 2.14(i) that $a b \in I$. Thus $\operatorname{diam}\left(\Gamma_{I}(R)\right)=1$.

Now assume that $\operatorname{diam}\left(\Gamma_{I}(R)\right)=2$. Let $\left.a / s, b / t \in \Gamma_{S^{-1} I}\left(S^{-1} R\right)\right)$. If $(a / s)(b / t) \notin S^{-1} I$, then $a b \notin I$, so there exists $c \in \Gamma_{I}(R)$ such that $a c \in I$ and $b c \in I$, so $c / 1 \in \Gamma_{S^{-1} I}\left(S^{-1} R\right)$ by Proposition 2.15. As $(a / s)(c / 1) \in S^{-1} I$ and $(c / 1)(b / t) \in S^{-1} I$, we must have $\operatorname{diam}\left(\Gamma_{S^{-1} I}\left(S^{-1} R\right)\right)=2$. Conversely, assume that $\operatorname{diam}\left(\Gamma_{S^{-1} I}\left(S^{-1} R\right)\right)=2$. Let $a, b \in \Gamma_{I}(R)$ with $a \neq b$. If $a b \notin I$, then $a b / 1 \notin S^{-1} I$ by Proposition 2.14(i), so there is an element $c / s$ of $\Gamma_{S^{-1} I}\left(S^{-1} R\right)$ with $(a / 1)(c / s) \in S^{-1} I$ and $(c / s)(b / 1) \in S^{-1} I$. In this case, by Proposition 2.15, we must have $c \in \Gamma_{I}(R)$. Moreover, Proposition 2.14(i) gives $a c \in I$ and $c b \in I$; hence $\operatorname{diam}\left(\Gamma_{I}(R)\right)=2$. Since, in general, the diameter of every zero-divisor graph with respect to an ideal is at most 3 , we have proved the result.

Example 2.17. Let $R=\mathbb{Z}$ and $I=6 \mathbb{Z}$. Then $I$ is not a primal ideal of $R$. Since 2 and 3 are not prime to $I$, but $3-2=1$ is prime to $I$. since $I=2 \mathbb{Z} \cap 3 \mathbb{Z}$, $\Gamma_{I}(R)$ is a complete bipartite with the parts $2 \mathbb{Z}-3 \mathbb{Z}$ and $3 \mathbb{Z}-2 \mathbb{Z}$ by $[11$, Theorem 3.1]. Thus diam $\left(\Gamma_{I}(R)\right)=2$. Set $S=\left\{3^{n}: n\right.$ is a non-negative integer $\}$. Then $S$ is a multiplicatively closed subset of $R$ whose elements are regular and $S^{-1} I$ is a prime ideal of $S^{-1} R$; hence $\Gamma_{S^{-1} I}\left(S^{-1} R\right)=\emptyset$. This example shows that the condition " $I$ is primal" in Theorem 2.16 is not superficial.

Theorem 2.18. Assume that $S$ is a multiplicatively closed subset of a ring $R$ which consists of regular elements of $R$ and let $I$ be a $P$-primal ideal of $R$ with $P \cap S=\emptyset$. Then $\operatorname{gr}\left(\Gamma_{I}(R)\right)=\operatorname{gr}\left(\Gamma_{S^{-1} I}\left(S^{-1} R\right)\right)$.

Proof. First assume that $\operatorname{gr}\left(\Gamma_{I}(R)\right)=\infty$. If $\operatorname{gr}\left(\Gamma_{S^{-1} I}\left(S^{-1} R\right)\right)=n$, then there is a cycle $a_{1} / s_{1}-a_{2} / s_{2}-\cdots-a_{n} / s_{n}$ in $\Gamma_{S^{-1} I}\left(S^{-1} R\right)$. In this case $a_{1}-a_{2}-$ $\cdots-a_{n}$ forms a cycle in $\Gamma_{I}(R)$ by Proposition 2.14(i) which is a contradiction. So $\operatorname{gr}\left(\Gamma_{S^{-1} I}\left(S^{-1} R\right)\right)=\infty$. If $\operatorname{gr}\left(\Gamma_{S^{-1} I}\left(S^{-1} R\right)\right)=\infty$, then since $\Gamma_{I}(R)$ is a subgraph of $\Gamma_{S^{-1} I}\left(S^{-1} R\right)$, we must have $\operatorname{gr}\left(\Gamma_{I}(R)\right)=\infty$. By [13, Lemma 5.1], 
the girth of every ideal-based zero-divisor graph of a commutative ring, when finite, is either 3 or 4 . Assume that $\operatorname{gr}\left(\Gamma_{S^{-1} I}\left(S^{-1} R\right)\right)=3$. So there exist distinct vertices $a / s, b / t, c / u$ in $\Gamma_{S^{-1} I}\left(S^{-1} R\right)$ such that $(a / s)(b / t),(b / t)(c / u)$ and $(c / u)(a / s)$ are elements of $S^{-1} I$, so Proposition 2.15 gives $a, b, c$ are distinct vertices of $\Gamma_{I}(R)$; hence $a b, b c, c a \in I$ by Proposition 2.14(i). It follows that $\operatorname{gr}\left(\Gamma_{I}(R)\right)=3$. Conversely, assume that $\operatorname{gr}\left(\Gamma_{I}(R)\right)=3$. Since the canonical homomorphism $R \rightarrow S^{-1} R$ is injective, we can assume that $R$ is a subring of $S^{-1} R$, so in this case, $\Gamma_{I}(R)$ is a subgraph of $\Gamma_{S^{-1} I}\left(S^{-1} R\right.$ ) (for if $a \in$ $\Gamma_{I}(R)$, then $\left.a / 1 \in S^{-1}\left(\Gamma_{I}(R)\right)=\Gamma_{S^{-1} I}\left(S^{-1} R\right)\right)$ by Proposition 2.15$)$; hence $\operatorname{gr}\left(\Gamma_{S^{-1} I}\left(S^{-1} R\right)\right) \leq \operatorname{gr}\left(\Gamma_{I}(R)\right)=3$. Since the girth of a graph is at least 3 , we must have $\operatorname{gr}\left(\Gamma_{S^{-1} I}\left(S^{-1} R\right)\right)=3$. Now it is clear that $\operatorname{gr}\left(\Gamma_{S^{-1} I}\left(S^{-1} R\right)\right)=4$ if and only if $\operatorname{gr}\left(\Gamma_{I}(R)\right)=4$.

\section{Non-primal ideals}

In this section we study the diameter of $\Gamma_{I}(R)$ where $I$ is not a primal ideal. First, we will give the following definition.

Definition. Let $I$ be an ideal of a ring $R$. An ideal $J$ of $R$ is called prime to $I$ if $\left(I:_{R} J\right)=I$.

Proposition 3.1. Let $I$ be an ideal of a ring $R$. If there are nonadjacent elements $a, b \in \Gamma_{I}(R)$ such that the ideal $\langle a, b\rangle$ is prime to $I$, then $\operatorname{diam}\left(\Gamma_{I}(R)\right)$ $=3$.

Proof. Since $a$ and $b$ are nonadjacent, we must have $\mathrm{d}(a, b) \neq 1$. If $\mathrm{d}(a, b)=2$, then there is an element $c \in R-I$ such that $a c, c b \in I$, so $c \in(I:\langle a, b\rangle)$ which is a contradiction. Thus $\mathrm{d}(a, b) \neq 2$, as required.

Proposition 3.2. Let $I$ be an ideal of a ring $R$. If $I$ is not primal, there exist elements $a$ and $b$ of $\Gamma_{I}(R)$ such that the ideal $\langle a, b\rangle$ is prime to $I$.

Proof. Suppose that $I$ is not primal. Then by Lemma 2.1, $\Gamma_{I}(R) \cup I=S(I)$ is not an ideal of $R$, so there exist $a, b \in S(I)$ with $a-b \notin S(I)$. If $a, b \in I$, then $a-b \in I \subseteq S(I)$ by Lemma 2.1 which is a contradiction. So suppose that $a \in I$ but $b \notin I$. Then $b \in \Gamma_{I}(R)$ and $b c \in I$ for some $c \in R-I$, so $(a-b) c \in I$; hence $a-b$ is not prime $I$ which is a contradiction. Similarly, for $a \notin I$ and $b \in I$, we get a contradiction. Thus, we must have $a, b \in R-I$, so $a, b \in \Gamma_{I}(R)$. It suffices to show that $(I:\langle a, b\rangle) \subseteq I$. If $r \in(I:\langle a, b\rangle)$, then $r(a-b) \in I$, so $r \in I$ since $a-b$ is prime to $I$. Thus $\langle a, b\rangle$ is prime to $I$.

Theorem 3.3. Let $I$ be a radical ideal of a ring $R$ and suppose that $I$ is not a primal ideal of $R$ and $|\operatorname{Min}(I)| \geq 3$. Then $\operatorname{diam}\left(\Gamma_{I}(R)\right)=3$.

Proof. By Proposition 3.2, there exist $a, b \in \Gamma_{I}(R)$ such that the ideal $\langle a, b\rangle$ is prime to $I$, so the ideal $\langle a+I, b+I\rangle$ of $R / I$ has no non-zero annihilator. As $R / I$ is a reduced ring and $|\operatorname{Min}(R / I)| \geq 3$, it follows from ([10, Theorem 2.1]) that $\operatorname{diam}(\Gamma(R / I))=3$. Now the assertion follows from Lemma 2.12. 
Theorem 3.4. Assume that $I$ is a radical ideal of a ring $R$ and $I$ is not a primal ideal of $R$. Then $\operatorname{diam}\left(\Gamma_{I}(R)\right) \leq 2$ if and only if $|\operatorname{Min}(I)|=2$.

Proof. First, assume that $\operatorname{diam}\left(\Gamma_{I}(R)\right) \leq 2$. By assumption, $S(I)$ is not an ideal of $R$, so there exist $a, b \in S(I)$ such that $a+b \notin S(I)$. If there is an element $r$ of $(I:\langle a, b\rangle)$ with $r \notin I$, then $a+b \in \Gamma_{I}(R) \subseteq S(I)$ which is a contradiction, so we must have $\langle a, b\rangle$ is prime to $I$. Therefore, $I$ is a radical ideal gives $I$ has at least two minimal prime ideals by Lemma 2.1(iii). If $I$ has more than two minimal primes, then $\operatorname{diam}\left(\Gamma_{I}(R)\right)=3$ by Theorem 3.3 ; hence $I$ must have exactly two minimal prime ideals. Next, assume that $|\operatorname{Min}(I)|=2$. If $P_{1}$ and $P_{2}$ are the only minimal prime ideals of $I$, then $S(I)=P_{1} \cup P_{2}$ by Lemma 2.1(iii) and we may assume $a \in P_{1}-P_{2}$ and $b \in P_{2}-P_{1}$. Clearly, $a b \in P_{1} \cap P_{2}=I$. Consider two distinct vertices $x$ and $y$ in $\Gamma_{I}(R)$. If $x y \in I$, then $\mathrm{d}(x, y)=1$. On the other hand, if $x y \notin I$, then either $\langle x, y\rangle \subseteq P_{1}$ or $\langle x, y\rangle \subseteq P_{2}$ since $S(I)=P_{1} \cup P_{2}$. If $\langle x, y\rangle \subseteq P_{1}$, then $x-b-y$ is a path in $\Gamma_{I}(R)$; hence $\mathrm{d}(x, y)=2$. A similar argument shows that if $\langle x, y\rangle \subseteq P_{2}$, then $\mathrm{d}(x, y)=2$. It follows that $\operatorname{diam}\left(\Gamma_{I}(R)\right) \leq 2$.

Lemma 3.5. Let $I$ be an ideal of a ring $R$ which is not a radical ideal, and let $J$ be an ideal of $R$ which is not prime to I. If $z \in \sqrt{I}$, then the ideal $R z+J$ is not prime to $I$.

Proof. By hypothesis, $S=R / I$ is a non-reduced ring and

$$
\left(0:_{R / I}(I+J) / I\right) \neq 0 .
$$

If $z \in \sqrt{I}$, then $z+I$ is a nilpotent element of $S$. It follows from [10, Lemma 2.3] that there exists a non-zero element $r+I$ in the annihilator of the ideal $S(z+$ $I)+(I+J) / I$ of $S$; hence $r \in\left(I:_{R} R z+J\right)-I$, as required.

Proposition 3.6. Assume that $I$ is not a radical ideal of a ring $R$. If $z \in \sqrt{I}$ and $a \in \Gamma_{I}(R)$, then $a+z \in \Gamma_{I}(R)$ and the ideal $\langle a, z\rangle$ is not prime to $I$.

Proof. This follows from Lemma 3.5.

Theorem 3.7. Let $I$ be an ideal of a ring $R$ which is not a radical ideal and suppose that $I$ is not a primal ideal of $R$. Then $\operatorname{diam}\left(\Gamma_{I}(R)\right)=3$.

Proof. By Proposition 3.2, there are elements $a, b \in \Gamma_{I}(R)$ such that the ideal $\langle a, b\rangle$ is prime to $I$, so $\mathrm{d}(a, b) \neq 2$. By Proposition 3.6, neither $a$ nor $b$ can be elements of $\sqrt{I}$. If $a b \notin I$, then $\mathrm{d}(a, b) \neq 1$, so d $(a, b)=3$; hence $\operatorname{diam}\left(\Gamma_{I}(R)\right)=$ 3 . So we can assume that $a b \in I$. Then

$$
\left(I:\left\langle a^{2}, b^{2}\right\rangle\right)=\left(I:\left\langle a^{2}, a b, b^{2}\right\rangle\right)=\left(I:\langle a, b\rangle^{2}\right)=(I:\langle a, b\rangle)=I .
$$

Therefore, there is an element $z \in \sqrt{I}$ such that $z \notin\left(I:\left\langle a^{2}, b^{2}\right\rangle\right)$. Without loss of generality we may assume that $z b^{2} \notin I$. By assumption and Proposition 3.6, we must have $a+b z \in \Gamma_{I}(R)$. Since $(I:\langle a+b z, b\rangle)=(I:\langle a, b\rangle)=I$, we get $\mathrm{d}(a+b z, b) \neq 2$. But $(a+b z) b=a b+b^{2} z \notin I$, so $\mathrm{d}(a+b z, b) \neq 1$. Thus $\mathrm{d}(a+b z, b)=3$ and $\operatorname{diam}\left(\Gamma_{I}(R)\right)=3$. 


\section{Weakly primal ideals}

In this section we study the ideal-based zero-divisor graph with respect to weakly prime and weakly primal ideals.

Theorem 4.1. Let $R$ be a finite local ring with unique maximal ideal $M$. Then $\Gamma(R)$ is a complete graph if and only if every proper ideal of $R$ is weakly prime.

Proof. Assume that $\Gamma(R)$ is a complete graph. Then $\Gamma(R)=M-\{0\}$. It then follows from $\left[2\right.$, Theorem 2.8] that $x y=0$ for all $x, y \in \Gamma(R)$, so $M^{2}=0$; hence the result follows from [3, Theorem 8]. Conversely, assume that every proper ideal of $R$ is weakly prime. Then by [3, Theorem 8], we must have $M^{2}=0$. Now the assertion follows from [2, Theorem 2.8].

Let $I$ be an ideal of a ring $R$. Set

$$
Z_{I}(R)=\{r \in R-I: r a=0 \text { for some } a \in R-I\} .
$$

Lemma 4.2. Let $I$ be a $P$-weakly primal ideal of a ring $R$. Then $\Gamma_{I}(R)=$ $(P-I) \cup Z_{I}(R)$.

Proof. Assume that $I$ is a $P$-weakly primal ideal of $R$ and let $r \in \Gamma_{I}(R)$. Then there is an element $a \in R-I$ with $r a \in I$. If $r a \neq 0$, then $r$ is not weakly prime to $I$, and so $r \in P-I$. If $r a=0$, then $r \in Z_{I}(R)$. So $\Gamma_{I}(R) \subseteq(P-I) \cup Z_{I}(R)$. For the reverse containment, assume that $s \in(P-I) \cup Z_{I}(R)$. If $s \in P-I$, then $s$ is not weakly prime to $I$, so $0 \neq s b \in I$ for some $b \in R-I$; hence $s \in \Gamma_{I}(R)$. If $s \in Z_{I}(R)$, then there is an element $c \in R-I$ such that $s c=0 \in I$; hence $s \in \Gamma_{I}(R)$, so we have equality.

Theorem 4.3. Let $I$ be $a$ an ideal of a ring $R$ and let $P$ be a weakly prime ideal of $R$ with $w(I) \subseteq P$ and $(P-I) \cap Z_{I}(R)=\emptyset$. Then $\Gamma_{I}(R)=(P-I) \cup Z_{I}(R)$ if and only if $I$ is a $P$-weakly primal ideal of $R$.

Proof. By Lemma 4.2, it suffices to show that if $\Gamma_{I}(R)=(P-I) \cup Z_{I}(R)$, then $I$ is a $P$-weakly primal ideal of $R$. We show that $P-\{0\}$ consists exactly of elements of $R$ that are not weakly prime to $I$. If $r \in R$ is not weakly prime to $I$, then $r \in w(I) \subseteq P$. Next, assume that $s \in P-\{0\}$. Since every non-zero element of $I$ is not weakly prime to $I$, we can assume that $s \notin I$. Therefore, $s \in P-I \subseteq \Gamma_{I}(R)$ implies that $s b \in I$ for some $b \in R-I$. Since $(P-I) \cap Z_{I}(R)=\emptyset$, we must have $s b \neq 0$; hence $s$ is not weakly prime to $I$. Thus $I$ is a $P$-weakly primal ideal of $R$.

Set $R=\mathbb{Z} / 24 \mathbb{Z}, I=8 \mathbb{Z} / 24 \mathbb{Z}$ and $P=2 \mathbb{Z} / 24 \mathbb{Z}$. Then $I$ is a $P$-primal ideal of $R$. Hence, by Proposition $2.2, \Gamma_{I}(R)=P-I=\{\overline{2}, \overline{4}, \overline{6}, \overline{10}, \overline{12}, \overline{14}, \overline{18}, \overline{20}, \overline{22}\}$. It is easy to check that $\Gamma_{I}(R)=Z_{I}(R)$. While $I$ is not a weakly prime ideal of $R$ because $\overline{0} \neq \overline{2} . \overline{4} \in I$ with $\overline{2}, \overline{4} \notin I$. This example shows that if $\Gamma_{I}(R)=Z_{I}(R)$, $I$ need not necessarily be weakly prime. But the converse holds:

Theorem 4.4. Let $I$ be a weakly prime ideal of a commutative ring $R$. Then $\Gamma_{I}(R)=Z_{I}(R)$. In particular, $\Gamma_{I}(R)$ is a subgraph of $\Gamma(R)$. 
Proof. Suppose that $I$ is a weakly prime ideal of $R$. Then $I$ is $I$-weakly primal by $\left[7\right.$, Theorem 3], so Lemma 4.2 gives $\Gamma_{I}(R)=Z_{I}(R)$.

The proof of the following corollary can be found in [7, Proposition 18], but our proof here will be different.

Corollary 4.5. Let I be an ideal of an integral domain $R$. Then $I$ is primal if and only if it is weakly primal.

Proof. Clearly, $Z_{I}(R)=\emptyset$. Now the assertion follows from Proposition 2.2 and Theorem 4.3.

Proposition 4.6. Assume that $S$ is a multiplicatively closed subset of a ring $R$ and let $I$ be a $P$-weakly primal ideal of $R$ with $P \cap S=\emptyset$. Then $S^{-1}\left(\Gamma_{I}(R)\right)=$ $\Gamma_{S^{-1} I}\left(S^{-1} R\right)$.

Proof. By [7, Proposition 9], $S^{-1} I$ is a $S^{-1} P$-weakly primal ideal of $S^{-1} R$. Then Lemma 4.2 gives

$$
\Gamma_{S^{-1} I}\left(S^{-1} R\right)=\left(S^{-1} P-S^{-1} I\right) \cup\left(Z_{S^{-1} I}\left(S^{-1} R\right)\right) .
$$

It is enough to show that

$$
S^{-1}\left[(P-I) \cup Z_{I}(R)\right]=\left(S^{-1} P-S^{-1} I\right) \cup\left(Z_{S^{-1} I}\left(S^{-1} R\right)\right) .
$$

First, assume that $a / s \in\left(S^{-1} P-S^{-1} I\right) \cup\left(Z_{S^{-1} I}\left(S^{-1}(R)\right.\right.$. If

$$
a / s \in Z_{S^{-1} I}\left(S^{-1} R\right),
$$

then $(a / s)(b / t)=a b / s t=0 \in S^{-1} I$ for some $b / t \in S^{-1}(R)-S^{-1} I$, so $b \notin I$ and $a b=0 \in I$; hence $a \in(P-I) \cup Z_{I}(R)$. Therefore, $a / s \in S^{-1}\left[(P-I) \cup Z_{I}(R)\right]$. If $a / s \in\left(S^{-1} P-S^{-1} I\right)$, then $a / s \notin S^{-1} I$ (so $\left.a \notin I\right)$ and $(a / s)(b / t)=a b / s t \in$ $S^{-1} I$ for some $b / t \notin S^{-1} I$ (so $b \notin I$ ). If $a b / s t=0$, then $a b=0 \in I$. If $a b / s t \neq 0$, then $a b \in I$ by [7, Lemma 8]. It follows that $a \in(P-I) \cup Z_{I}(R)$. Thus $\left(S^{-1} P-S^{-1} I\right) \cup\left(Z_{S^{-1} I}\left(S^{-1} R\right) \subseteq S^{-1}\left[(P-I) \cup Z_{I}(R)\right]\right.$. Next, suppose that $a / s \in S^{-1}\left[(P-I) \cup Z_{I}(R)\right]$. Then $a b \in I$ for some $b \notin I$. By [7, Lemma 8], $b / 1 \notin S^{-1} I$. Clearly, $(a / s)(b / 1)=a b / s \in S^{-1} I$. If $(a / s)(b / 1)=0$, then $\left.a / s \in Z_{S^{-1} I}\left(S^{-1} R\right)\right)$. If $(a / s)(b / 1) \notin 0$, then $a / s$ is not weakly prime to $S^{-1} I$, so $a / s \in S^{-1} P-S^{-1} I$; hence $S^{-1}\left[(P-I) \cup Z_{I}(R)\right] \subseteq\left(S^{-1} P-S^{-1} I\right) \cup$ $\left(Z_{S^{-1} I}\left(S^{-1} R\right)\right)$, so the proof is complete.

Theorem 4.7. Assume that $S$ is a multiplicatively closed subset of a ring $R$ which consists of regular elements of $R$ and let $I$ be a $P$-weakly primal ideal of $R$ with $P \cap S=\emptyset$. Then $\operatorname{diam}\left(\Gamma_{I}(R)\right)=\operatorname{diam}\left(\Gamma_{S^{-1} I}\left(S^{-1} R\right)\right)$.

Proof. Suppose that $\operatorname{diam}\left(\Gamma_{I}(R)\right)=1$. For every distinct vertices $a / s, b / t$ of $\Gamma_{S^{-1} I}\left(S^{-1} R\right)$ ), Proposition 4.6 gives $a$ and $b$ are distinct elements of $\Gamma_{I}(R)$, so $a b \in I$; hence $(a / s)(b / t) \in S^{-1} I$. Thus $\operatorname{diam}\left(\Gamma_{S^{-1} I}\left(S^{-1} R\right)\right)=1$. If $\operatorname{diam}\left(\Gamma_{S^{-1} I}\left(S^{-1} R\right)\right)=1$, then for every distinct vertices $a, b$ of $\Gamma_{I}(R)=$ $(P-I) \cup Z_{I}(R)$, we must have $a / 1, b / 1 \in S^{-1}\left((P-I) \cup Z_{I}(R)\right)=\Gamma_{S^{-1} I}\left(S^{-1} R\right)$ 
by Proposition 4.6, so $(a / 1)(b / 1) \in S^{-1} I$. If $a b=0$, then $a b \in I$. If $a b \neq 0$, then [7, Lemma 8] gives $a b \in I$. Thus $\operatorname{diam}\left(\Gamma_{I}(R)\right)=1$.

The proof of the cases when diameters are 2 and 3 is similar to that in case $\operatorname{diam}\left(\Gamma_{I}(R)\right)=1$ and Theorem 2.16, and we omit it.

Theorem 4.8. Assume that $S$ is a multiplicatively closed subset of a ring $R$ which consists of regular elements of $R$ and let $I$ be a $P$-weakly primal ideal of $R$ with $P \cap S=\emptyset$. Then $\operatorname{gr}\left(\Gamma_{I}(R)\right)=\operatorname{gr}\left(\Gamma_{S^{-1} I}\left(S^{-1} R\right)\right)$.

Proof. By [13, Theorem 5.5], the girth of every ideal-based zero-divisor graph of a commutative ring, when finite, is either 3 or 4 . By using Proposition 4.6 and [7, Lemma 8$]$ the proof is similar to that in the Theorem 2.18 and we omit it.

Theorem 4.9. Assume that $S$ is a multiplicatively closed subset of a ring $R$ which consists of regular elements of $R$ and let $P$ be a weakly prime ideal of $R$ with $P \cap S=\emptyset$. Then the following hold:

(i) $\operatorname{diam}\left(\Gamma_{P}(R)\right)=\operatorname{diam}\left(\Gamma_{S^{-1} P}\left(S^{-1} R\right)\right)$.

(ii) $\operatorname{gr}\left(\Gamma_{P}(R)\right)=\operatorname{gr}\left(\Gamma_{S^{-1} P}\left(S^{-1} R\right)\right)$.

Proof. By [7, Theorem 3], every weakly prime ideal of $R$ is weakly primal. Also, $S^{-1} P$ is weakly prime ideal of $S^{-1} R$ by [3, Proposition 13]. Now the assertion follows from Theorem 4.7 and Theorem 4.8.

Let $Q(R)$ be the total quotient ring of $R$. It is proved in [1, Theorem 2.2] (see also $[12$, Theorem 1.1]) that the graphs $\Gamma(R)$ and $\Gamma(Q(R))$ are isomorphic. So these two graphs have the same diameters and same girths. This theorem is basic and several important results follows from it. Now consider the following remark:

Remark 4.10. Suppose that $Q(R)=T^{-1} R$ is the total quotient ring of $R$. We know that 0 always is a weakly prime ideal of $R$ (by definition). Therefore, by Theorem 4.9, $\operatorname{diam}(\Gamma(R))=\operatorname{diam}\left(\Gamma_{0}(R)\right)=\operatorname{diam}(\Gamma(Q(R))$. Thus Theorem 4.9 is a generalization of $[12$, Theorem 1.1].

Acknowledgments. The authors thank the referee for his/her excellent suggestions to an earlier version of the paper, which improved the paper.

\section{References}

[1] D. F. Anderson, R. Levy, and J. Shapirob, Zero-divisor graphs, von Neumann regular rings, and Boolean algebras, J. Pure Appl. Algebra 180 (2003), no. 3, 221-241.

[2] D. F. Anderson and P. S. Livingston, The zero-divisor graph of a commutative ring, J. Algebra 217 (1999), no. 2, 434-447.

[3] D. D. Anderson and E. Smith, Weakly prime ideals, Houston J. Math. 29 (2003), no. 4, 831-840.

[4] I. Beck, Coloring of commutative rings, J. Algebra 116 (1988), no. 1, 208-226.

[5] L. Dancheng and W. Tongsuo, On ideal-based zero-divisor graphs, Preprint 2006.

[6] S. Ebrahimi Atani and A. Yousefian Darani, Some remarks on primal submodules, Sarajevo Journal of Mathematics 4 (2008), no. 17, 181-190. 
[7] _ On weakly primal ideals (I), Demonstratio Math. 40 (2007), no. 1, 23-32.

[8] L. Fuchs, On primal ideals, Proc. Amer. Math. Soc. 1 (1950), 1-6.

[9] J. A. Huckaba, Commutative Rings with Zero Divisors, Monographs and Textbooks in Pure and Applied Mathematics, 117. Marcel Dekker, Inc., New York, 1988.

[10] T. G. Lucas, The diameter of a zero divisor graph, J. Algebra 301 (2006), no. 1, 174-193.

[11] H. R. Maimani, M. R. Pournaki, and S. Yassemi, Zero-divisor graph with respect to an ideal, Comm. Algebra 34 (2006), no. 3, 923-929.

[12] S. B. Mulay, Rings having zero-divisor graphs of small diameter or large girth, Bull. Austral. Math. Soc. 72 (2005), no. 3, 481-490.

[13] S. P. Redmond, An ideal-based zero-divisor graph of a commutative ring, Comm. Algebra 31 (2003), no. 9, 4425-4443.

[14] R. Y. Sharp, Steps in Commutative Algebra, London Mathematical Society Student Texts, 19. Cambridge University Press, Cambridge, 1990.

Shahabaddin Ebrahimi Atani

Department of Mathematics

Guilan UNIVERSITY

RASHT, IRAN

E-mail address: ebrahimi@guilan.ac.ir

Ahmad Yousefian Darani

Department of Mathematics

University of Mohaghegh Ardabili

P. O. Box 179, Ardabil, Iran

E-mail address: youseffian@gmail.com

or yousefian@uma.ac.ir 